\title{
Curcumin suppresses breast tumor angiogenesis by abrogating osteopontin-induced VEGF expression
}

\author{
GOUTAM CHAKRABORTY, SHALINI JAIN, SMITA KALE, REMYA RAJA, \\ SANTOSH KUMAR, ROSALIN MISHRA and GOPAL C. KUNDU \\ National Center for Cell Science, Pune, India
}

Received April 9, 2008; Accepted June 2, 2008

DOI: $10.3892 / \mathrm{mmr} 00000005$

\begin{abstract}
The development and progression of malignant tumors depends on the formation of new blood vessels inside the tumor. This phenomenon is termed tumor angiogenesis. Angiogenesis is one of the fundamental processes that occur during cancer progression, and depends on the expression and activation of various angiogenic molecules, cytokines, growth factors, kinases and transcription factors. We recently demonstrated that the chemokine-like ECM-associated protein osteopontin (OPN) turns on the angiogenic switch by upregulating expression of vascular endothelial growth factor (VEGF) in a human breast cancer model. Furthermore, we proposed that targeting OPN-induced VEGF expression could be a potential therapeutic approach for the treatment of breast cancer. In this study, we demonstrate that curcumin (diferuloylmethane) abrogates OPN-induced VEGF expression and curbs OPN-induced VEGF-dependent breast tumor angiogenesis in vivo. We also explore the fact that curcumin in combination with anti-VEGF or anti-neuropilin (NRP)-1 antibody exhibits enhanced anti-angiogenic activity compared to curcumin alone. Our results indicate that curcumin suppresses OPN-induced VEGF expression and tumor angiogenesis, and suggest that this study may aid in the development of a curcumin-based OPN-targeted therapeutic approach to the control of breast tumor angiogenesis.
\end{abstract}

\section{Introduction}

Breast cancer is considered to be one of the most common cancer threats worldwide. According to the World Health Organization (WHO), it is the most deadly cancer in females, with more than 500,000 deaths attributed to it globally each year. To date, the major cause of breast cancer is not clearly understood. However, experimental evidence has revealed that the expression profiles of certain oncogenic molecules or

Correspondence to: Dr Gopal C. Kundu, National Center for Cell Science, Pune 411 007, India

E-mail: kundu@nccs.res.in

Key words: osteopontin, vascular endothelial growth factor, curcumin, human breast cancer, tumor angiogenesis biomarkers are significantly associated with breast cancer progression, and have shown great promise in breast cancer therapy $(1,2)$. Osteopontin (OPN), a chemokine-like ECMassociated small integrin binding ligand $\mathrm{N}$-linked glycoprotein (SIBLING), has recently been identified as one of the major markers of breast cancer progression $(3,4)$. Elevated expression of OPN at tumor sites as well as in the serum of breast cancer patients has signalled the prognostic importance of this protein in breast cancer $(5,6)$. Moreover, the targeting of OPN and its downstream signaling pathways has shown great promise in the therapeutics of various cancers, breast cancer included (7). The role of OPN in breast cancer progression is therefore being intensely investigated.

Angiogenesis, or the formation of new blood vessels from existing ones, is a key step in tumor growth, survival, progression and metastasis (8). Tumor angiogenesis is thought to result from the secretion of 'angiogenetic factors' by tumor cells. These include growth factors, cytokines and a number of small molecules $(9,10)$. Of these, vascular endothelial growth factor (VEGF) has been recognized as the most important (11). Highly malignant tumors are characterized by enhanced vascularization, which is further correlated with elevated VEGF expression (12). Previous reports suggest that tumorderived VEGF interacts with tumor or endothelial cell surface receptors via autocrine or paracrine mechanisms and promotes tumor angiogenesis (13-15). We recently demonstrated that OPN augments VEGF expression and promotes VEGFdependent breast tumor angiogenesis (16). Therefore, we can hypothesize that targeting OPN-induced VEGF might serve as a potential therapeutic approach for the treatment of breast cancer.

Curcumin (diferuloylmethane) is a polyphenol derived from the rhizomes of Curcuma Longa, traditionally used as an anti-inflammatory compound, and appears to be useful in the prevention and treatment of various cancers (17-19). Curcumin has exhibited a significant inhibitory effect on several malignant cancers, including breast cancer (20). It has also been reported that curcumin downregulates the activation of NF- $\mathrm{\kappa B}$ and suppresses tumor growth in various cancer models (21-23). We have previously reported that curcumin suppressed OPNinduced MMP-2 activation and tumor growth in a murine melanoma model (24). However, the role of curcumin in the regulation of OPN-induced VEGF-dependent breast tumor angiogenesis is not well defined. In this study, we demonstrate that curcumin abrogates OPN-induced VEGF expression and suppresses VEGF-dependent breast tumor angiogenesis. 


\section{Materials and methods}

Cell culture. The human breast adenocarcinoma cell line MDA-MB-231 was obtained from American Type Culture Collection (Manassas, VA, USA). Cells were cultured in L-15 media supplemented with $10 \% \mathrm{FBS}, 100 \mu \mathrm{g} / \mathrm{ml}$ streptomycin and 100 units/ml penicillin. Human umbilical vein endothelial cells (HUVEC) were obtained from Lonza (Walkersville, MD, USA) and cultured in the EBM bullet kit (Lonza) according to the manufacturer's instructions.

Western blot analysis and EMSA. Western blot analysis and EMSA were performed as previously described $(16,25)$. Briefly, the cells were treated with $0.5 \mu \mathrm{M}$ OPN, or pretreated with various doses of curcumin $(0-50 \mu \mathrm{M})$ and then treated with OPN. Cell lysates were used to detect VEGF expression by Western blot analysis, and the nuclear extracts were used for NF- $\kappa$ B and ATF-4-DNA binding by EMSA.

Immunofluorescence study. Immunofluorescence staining was performed using a standard protocol as described previously (26). Briefly, the cells were treated with $0.5 \mu \mathrm{M}$ OPN, or pretreated with $20 \mu \mathrm{M}$ curcumin and then treated with OPN. Cells were fixed in $2 \%$ paraformaldehyde by incubation at $4^{\circ} \mathrm{C}$ for $15 \mathrm{~min}$. After quenching with $0.1 \%$ glycine, cells were permeabilized with $0.1 \%$ Triton $\mathrm{X}-100$, washed three times with PBS for $5 \mathrm{~min}$ and then incubated in 2\% BSA in PBS ( $\mathrm{pH} 7.4$ ) for $1 \mathrm{~h}$ at room temperature (RT) to block nonspecific binding. Subsequently, cells were incubated with specific primary antibody in $0.2 \% \mathrm{BSA}$ for $2 \mathrm{~h}$ at RT, followed by incubation with secondary antibody for $1 \mathrm{~h}$ at RT. Nuclei were stained with PI. Cells were washed and mounted in mounting media and analyzed using a confocal microscope (Zeiss).

Wound migration assay. The motility of MDA-MB-231 cells was determined by wound migration assay as previously described $(16,27)$. Cells were treated with $0.5 \mu \mathrm{M}$ OPN, or pretreated with 0-20 $\mu \mathrm{M}$ curcumin followed by treatment with OPN. In separate experiments, cells were pretreated with $10 \mu \mathrm{M}$ curcumin along with anti-VEGF or anti-neuropilin (NRP)-1 antibody and then treated with OPN. After $12 \mathrm{~h}$, wound photographs were captured using a phase contrast microscope (Nikon).

Co-migration assay. Tumor-endothelial cell interactions were carried out using a co-migration assay with a modified Boyden chamber. MDA-MB-231 cells were treated with $0.5 \mu \mathrm{M}$ OPN alone and used in the lower chamber. Endothelial cells (HUVEC) were seeded on the upper chamber. In separate experiments, MDA-MB-231 cells were pretreated with curcumin and then treated with OPN alone or with antiVEGF or anti-NRP-1 antibody in the lower chamber, and co-migration experiments were performed. The migrated endothelial cells were stained with Giemsa, photographed and then counted in three high-power fields under an inverted microscope (Nikon). They were then analyzed statistically, and the resulting data was represented by a bar graph.

In vivo Matrigel-based angiogenesis assay. The in vivo Matrigel angiogenesis assay was performed as previously described (16). Briefly, Matrigel (0.5 ml) was subcutaneously injected into the ventral groin region of female athymic NMRI $(\mathrm{nu} / \mathrm{nu})$ mice. In separate experiments, the conditioned medium (CM) of untreated or OPN-treated MDA-MB-231 cells was mixed with Matrigel and injected into the mice. In other experiments, OPN-treated CM was mixed with curcumin $(20 \mu \mathrm{M})$ in Matrigel and then injected into the mice. After 3 weeks, Matrigel plugs were excised and processed for histopathology and immunohistochemistry using specific antibodies.

Immunohistochemical study. Immunohistochemical analysis of paraffin-embedded sections was performed using standard procedure as previously described $(16,27)$. After deparaffinization in xylene, the sections were re-hydrated using descending grades of ethanol (100, 95 and 70\%), washed thrice in PBS, then used for antigen retrieval. After quenching with $100 \mathrm{mM}$ glycine, tissue sections were permeabilized with $0.1 \%$ Triton $\mathrm{X}-100$, washed twice with PBS, then blocked with $2 \%$ bovine serum albumin for $1 \mathrm{~h}$. Finally, the sections were incubated with specific primary antibodies overnight at $4^{\circ} \mathrm{C}$. Subsequently, the slides were washed in PBS and incubated with specific secondary antibodies for $2 \mathrm{~h}$. The sections were mounted and analyzed using a confocal microscope (Zeiss).

Statistical analysis. Bands were analyzed densitometrically (Kodak Digital Science) and fold changes were calculated. The wound migration and co-migration assays were analyzed statistically, and the data were represented by means of a bar graph. Statistical differences were determined by the paired Student's t-test. Differences were considered significant at P-values $<0.05$.

\section{Results}

Curcumin abrogates OPN-induced VEGF expression. We previously reported that curcumin suppressed OPN-induced MMP-2 activation in a murine melanoma model (24). More recently, we demonstrated that OPN augments VEGF expression in human breast cancer cells (16). To examine the effect of curcumin on OPN-induced VEGF expression, serum-starved MDA-MB-231 cells were either treated with $0.5 \mu \mathrm{M}$ OPN or were pretreated with curcumin and then treated with OPN. The results indicate that curcumin significantly abrogates OPNinduced VEGF expression in these cells, with maximum inhibition observed using $20 \mu \mathrm{M}$ of curcumin (Fig. 1A).

Effects of curcumin on $O P N$-induced $N F-\kappa B$ and ATF-4-DNA binding. We recently showed that OPN promotes VEGF expression via activation of NF- $\mathrm{KB}$ in MDA-MB-231 cells (16). However, it has been reported that curcumin suppresses $\mathrm{NF}-\kappa \mathrm{B}$ activation in various cancer cells (21-23). Therefore, to examine whether curcumin inhibits OPN-induced NF-кBDNA binding, cells were pretreated with curcumin and then treated with OPN, and EMSA was performed. The data showed that curcumin significantly suppressed OPN-induced NF-кB-DNA binding (Fig. 1B).

The ATF-4 response element has been reported to be in the promoter region of VEGF $(28,29)$. ATF-4 plays a crucial role in OPN-induced VEGF expression (16). To confirm the effect of curcumin on OPN-induced ATF-4-DNA binding 
A

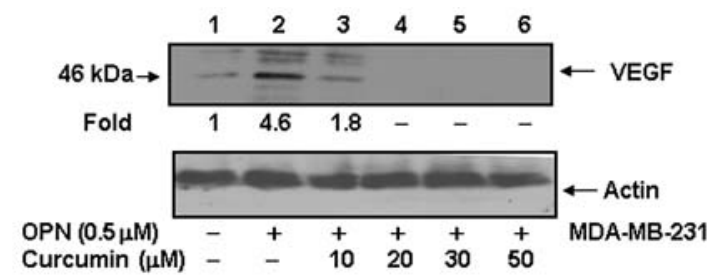

C

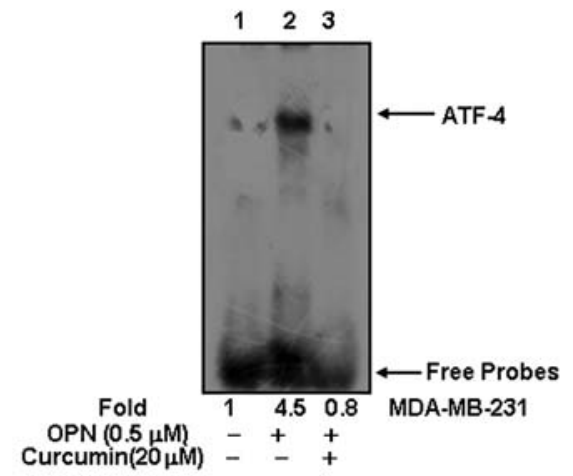

B
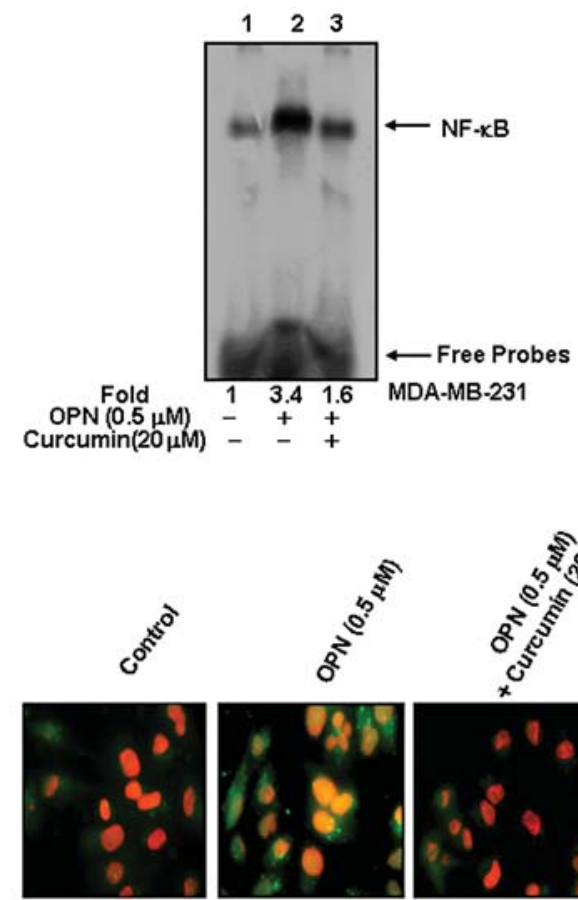

Figure 1. (A) Curcumin inhibits OPN-induced VEGF expression. MDA-MB-231 cells were treated with OPN for 6 h, or pretreated with the indicated concentrations of curcumin for $2 \mathrm{~h}$ and then treated with OPN. The level of VEGF in the cell lysate was determined by Western blot analysis. Actin was used as the control. (B-D) Curcumin suppresses OPN-induced NF- $\mathrm{BB}$ and ATF-4-DNA binding and ATF-4 nuclear localization. MDA-MB-231 cells were treated with OPN alone, or pretreated with curcumin followed by treatment with OPN. NF-кB and ATF-4-DNA binding activities were measured usng EMSA. Nuclear localization of ATF-4 was determined by immunofluorescence. ATF-4 was stained with Cy 2 (green), and nuclei were stained with PI (red).

A

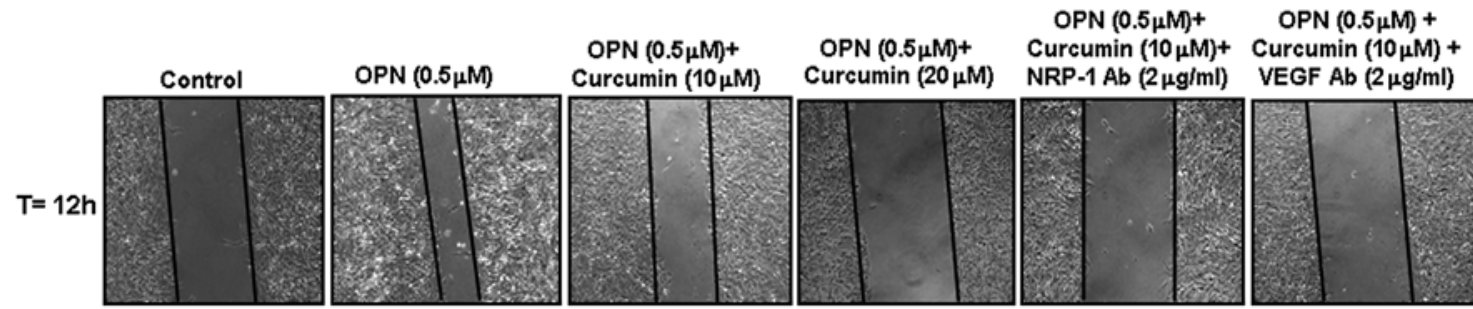

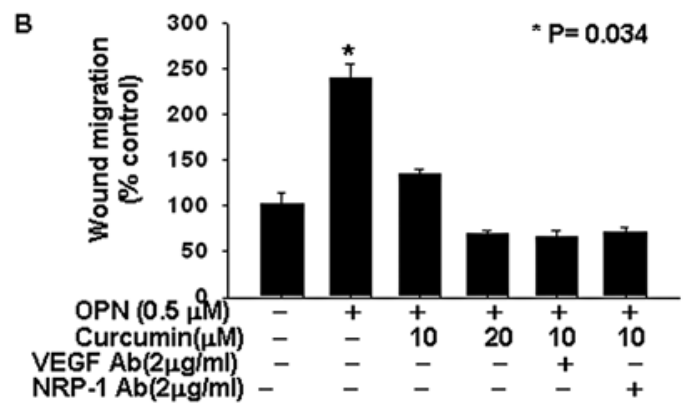

Figure 2. (A and B) Suppression of OPN-induced tumor cell motility by curcumin. MDA-MB-231 cells were pretreated with curcumin alone or in combination with anti-VEGF or anti-NRP-1 antibody, followed by treatment with OPN. A wound assay was performed and wound photographs were captured (A). Data are presented in the form of a bar graph (B). ${ }^{*} \mathrm{P}=0.034$

and nuclear localization, cells were pretreated with curcumin and then treated with OPN. Analysis of ATF-4-DNA binding and nuclear localization was performed by EMSA and immunofluorescence, respectively. The data indicate that curcumin inhibits OPN-induced ATF-4-DNA binding as well as nuclear localization (Fig. 1C and D). This suggests that curcumin curbs OPN-induced NF- $\mathrm{KB}$ and ATF-4 activation, and thereby alters OPN-induced VEGF expression in human breast cancer cells.

Curcumin inhibits OPN-induced VEGF-dependent breast tumor cell motility. To ascertain whether curcumin has any effect on OPN-induced breast tumor cell motility, a wound migration assay was performed using MDA-MB-231 cells. Cells were treated with OPN, or pretreated with curcumin alone or in combination with anti-VEGF or anti-NRP-1 antibody and then treated with OPN. Wound photographs were captured and analyzed, and the data represented by a bar graph (Fig. 2A and B). The data indicate that curcumin significantly inhibits OPN-induced tumor cell motility. A low concentration of curcumin in combination with anti-VEGF or anti-NRP-1 antibody also inhibited OPN-induced cell motility; however, the percent of inhibition was higher in this condition as compared to curcumin alone (Fig. 2A and B). These data suggest that curcumin suppresses OPN-induced VEGF/ NRP-1-dependent breast tumor cell motility. 


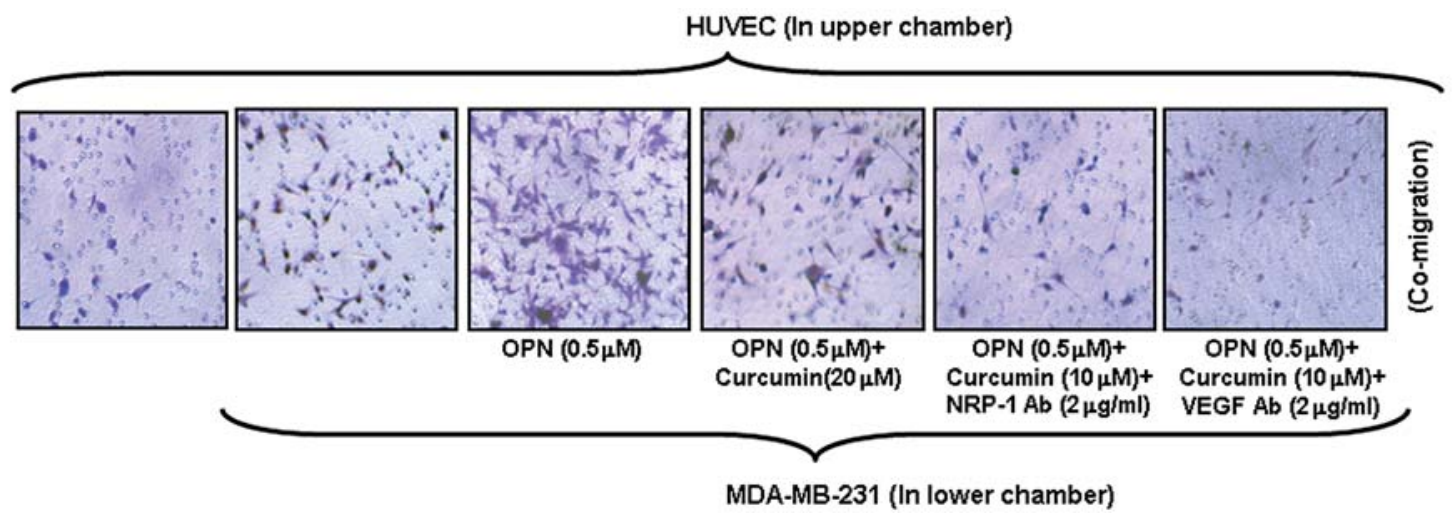

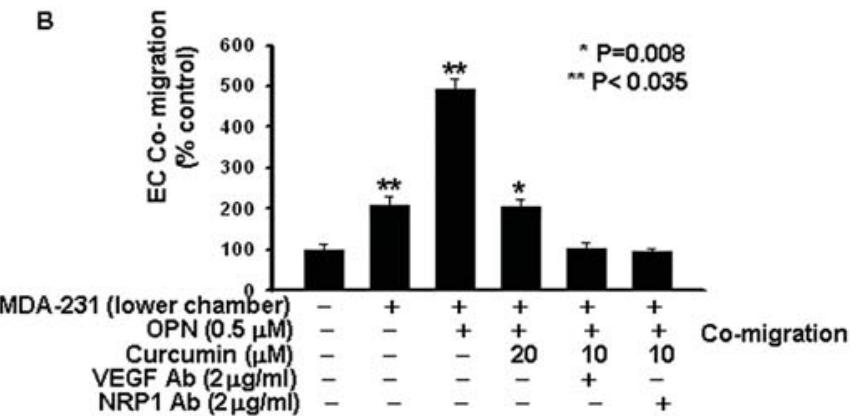

Curcumin suppresses OPN-induced VEGF-dependent tumorendothelial interaction. To examine the effect of curcumin on the suppression of OPN-induced tumor-endothelial cell interaction, a co-migration assay was performed using MDA-MB231 cells and HUVEC in a modified Boyden chamber. After termination of the experiments, HUVEC which had migrated from the upper to the lower chamber were stained with Giemsa, photographed, and counted in three high-power fields. The resulting data were represented by a bar graph (Fig. 3A and B). The data indicate that OPN-induced endothelial cell migration towards tumor cells was suppressed by curcumin (Fig. 3A and B). More importantly, curcumin with anti-VEGF or anti-NRP-1 antibody suppressed OPN-induced migration of endothelial cells towards tumor cells, further suggesting that OPN-induced tumor-derived VEGF promotes tumorendothelial interaction and that curcumin suppresses this effect.

Inhibition of $O P N$-induced in vivo Matrigel plug angiogenesis by curcumin. To determine the role of curcumin in the suppression of OPN-induced in vivo angiogenesis, a Matrigel plug angiogenesis assay was performed in nude mice. Conditioned medium (CM) collected from untreated or OPN-treated MDA-MB-231 cells was mixed with Matrigel and injected subcutaneously into the ventral groin region of female nude mice as described previously (16). In separate experiments, curcumin was mixed with the CM of OPN-treated MDA-MB231 cells along with Matrigel and then injected into mice. After 21 days, the mice were sacrificed and the Matrigel plugs photographed (Fig. 4, panel I). The data indicate that curcumin radically suppressed OPN-induced in vivo angiogenesis as compared to the controls (Fig. 4). The excised Matrigel plugs were analyzed histopathologically (panels II and III; magnification, x10 and x60) and by immunofluorescence using antivWF (Von Willebrand Factor, an endothelial cell marker) and anti-pKDR antibodies. The data indicate that curcumin significantly suppressed OPN-induced in vivo angiogenesis (panel IV) and KDR phosphorylation. Taken together, the data demonstrate that curcumin curtails $\mathrm{OPN}$-induced in vivo angiogenesis by inhibiting KDR phosphorylation.

\section{Discussion}

In the current study, we have demonstrated that curcumin inhibits OPN-induced VEGF expression, which in turn suppresses tumor and endothelial cell motility and in vivo angiogenesis. Previously, we reported that NF- $\mathrm{\kappa B}$ and ATF-4 play a crucial role in OPN-induced VEGF expression (16). In this study, we found that curcumin suppresses OPN-induced NF- $\kappa$ B and ATF-4-DNA binding and ATF-4 nuclear localization. Thus, curcumin abrogates OPN-induced VEGF expression by suppressing the binding activities of NF- $\mathrm{\kappa B}$ and ATF-4. Our data also indicate that OPN-induced VEGFdependent in vivo angiogenesis was downregulated by curcumin. This clearly suggests that curcumin acts as a potent anti-angiogenic agent in regulating OPN-induced tumor angiogenesis.

The role of OPN in tumor angiogenesis is being intensely investigated $(30,31)$. Previously, OPN was considered to be a metastasis-associated gene (32). Recently, however, its role in tumor angiogenesis has been demonstrated $(16,27,30)$. Angiogenesis-based therapy has emerged as one of the major therapeutic approaches to cancer (33). Thus, identifying novel therapeutic approaches to targeting the angiogenic pathways could prove to be promising for cancer therapy. We previously showed that OPN acts as a potent angiogenic molecule by regulating VEGF expression in a breast cancer model (16). Consequently, targeting OPN-regulated angiogenic pathways could be a promising therapeutic strategy for the treatment of breast cancer. 


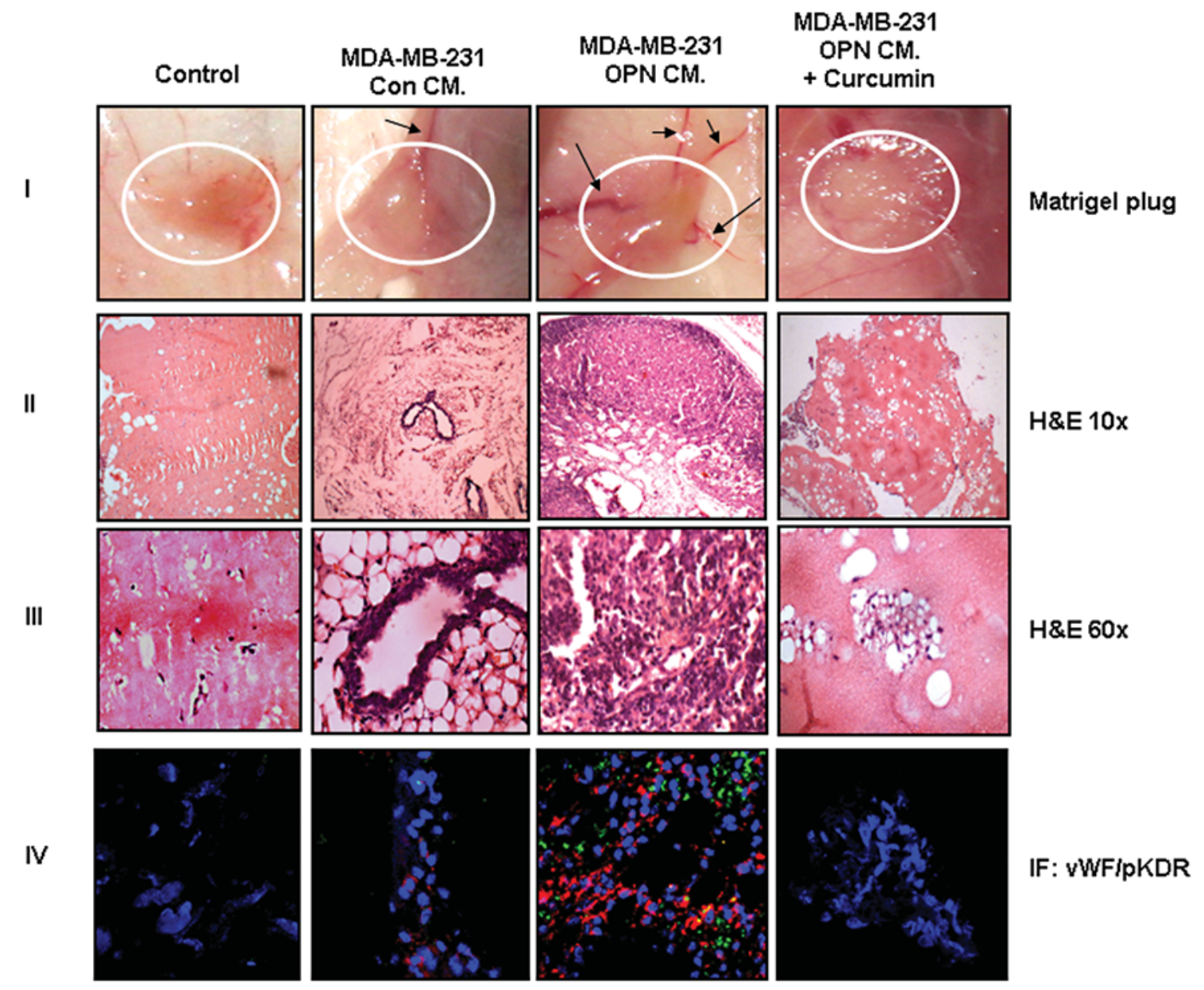

Figure 4. Curcumin abrogates OPN-induced in vivo tumor angiogenesis. Typical photographs of in vivo Matrigel plug angiogenesis in nude mice. Angiogenic blood vessels are indicated by arrows. Note the significant reduction in blood vessel formation in the plug treated with curcumin and OPN compared with OPN alone (panel I). Matrigel plugs were analyzed histopathologically by H\&E staining, and photographs were captured at a magnification of x10 and x60 (panels II and III). Matrigel plug sections were immunohistochemically analyzed using anti-vWF and anti-pKDR antibodies (panel IV). vWF was stained with Cy3 (red) and p-KDR with FITC (green). Nuclei were stained with DAPI (blue).

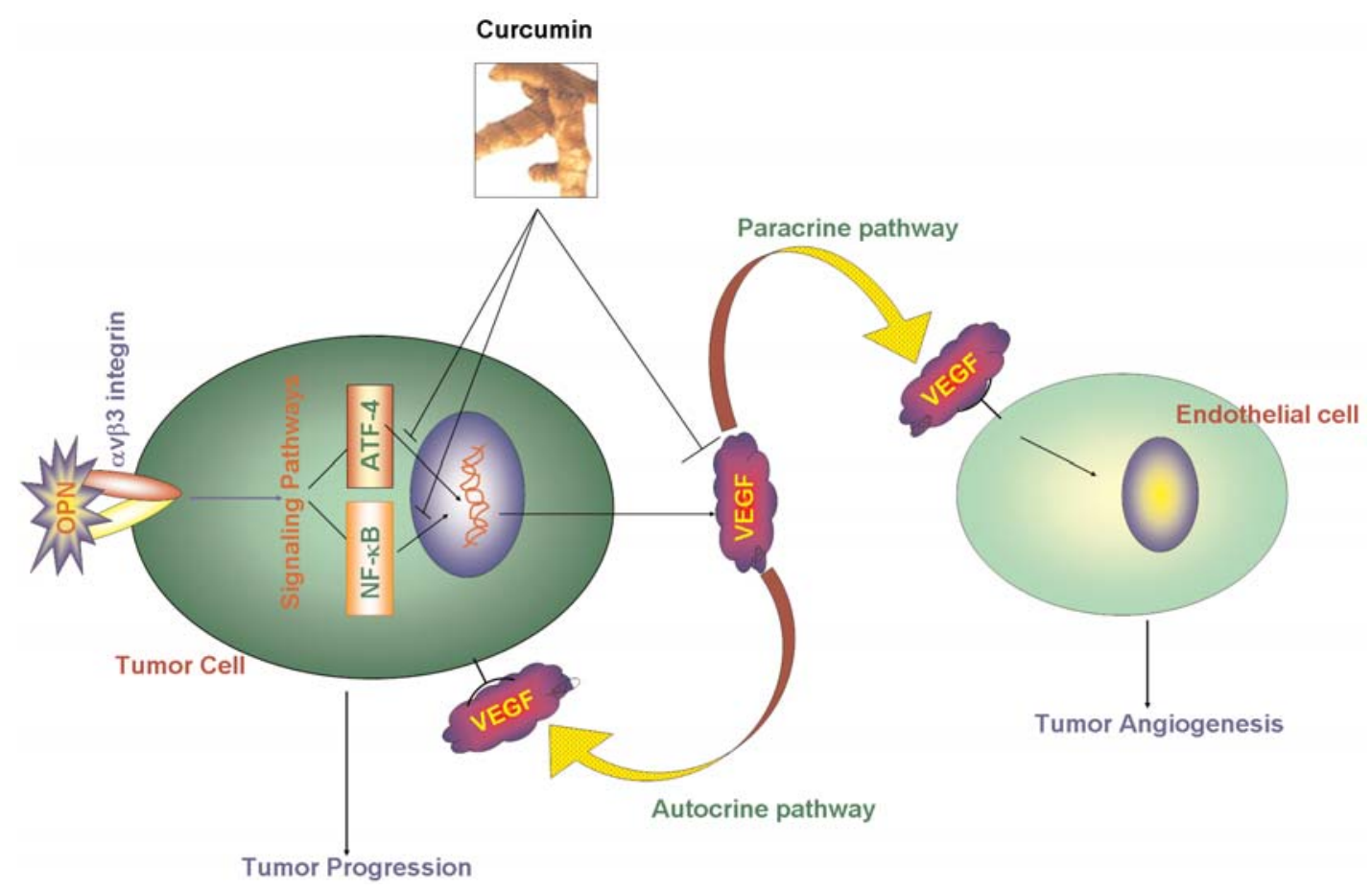

Figure 5. Schematic representation of the curcumin suppression of OPN-induced VEGF expression leading to the inhibition of tumor growth and angiogenesis. 
Curcumin has long been used as a potent anti-inflammatory agent. Recently, though, this molecule has been identified as having anti-cancer properties (17-19). Curcumin also inhibits PMA-induced VEGF mRNA expression and suppresses angiogenesis (19). Previously, we found that it suppressed OPNregulated murine melanoma growth by inhibiting MMP-2 activation (25). However, the role of curcumin in OPN-induced breast tumor angiogenesis is not well defined. In this study, we demonstrated that curcumin abrogates OPN-induced VEGF expression, thus inhibiting tumor angiogenesis. Moreover, anti-VEGF or anti-NRP-1 antibody along with curcumin resulted in higher anti-angiogenic activity than curcumin alone. We therefore hypothesize that curcumin along with antiVEGF or anti-NRP-1 antibody could be a novel approach for the treatment of cancer.

In this study, we have gone some way in demonstrating for the first time that curcumin inhibits OPN-induced NF- $\mathrm{NB}$ / ATF-4-dependent VEGF expression, ultimately suppressing breast tumor angiogenesis (Fig. 5). As OPN is considered to be a major molecule in the control of breast cancer progression, targeting the OPN-regulated signaling pathway by curcumin could be an emerging approach to the treatment of the disease. This approach may help block the OPN-regulated VEGFdependent angiogenic switch, and could also contribute to the development of a novel strategy for the treatment of breast cancer.

\section{Acknowledgements}

This work was supported by the Council of Scientific and Industrial Research, Government of India (to G.C. and S.J.).

\section{References}

1. Chatterjee SK and Zetter BR: Cancer biomarkers: knowing the present and predicting the future. Future Oncol 1: 37-50, 2005.

2. Payne SJ, Bowen RL, Jones JL and Wells CA: Predictive markers in breast cancer - the present. Histopathology 52: 82-90, 2008.

3. Rodrigues LR, Teixeira JA, Schmitt FL, Paulsson M and Lindmark-Mänsson $\mathrm{H}$ : The role of osteopontin in tumor progression and metastasis in breast cancer. Cancer Epidemiol Biomarkers Prev 16: 1087-1097, 2007.

4. Mirza M, Shaughnessy E, Hurley JK, Vanpatten KA, Pestano GA, $\mathrm{He} \mathrm{B}$ and Weber GF: Osteopontin-c is a selective marker of breast cancer. Int J Cancer 122: 889-897, 2008.

5. Rudland PS, Platt-Higgins A, El-Tanani M, De Silva Rudland S, Barraclough R, Winstanley JH, Howitt R and West CR: Prognostic significance of the metastasis-associated protein osteopontin in human breast cancer. Cancer Res 62: 3417-3427, 2002.

6. Tuck AB, Chambers AF and Allan AL: Osteopontin overexpression in breast cancer: knowledge gained and possible implications for clinical management. J Cell Biochem 102: 859-868, 2007.

7. Jain S, Chakraborty G, Bulbule A, Kaur R and Kundu GC: Osteopontin: an emerging therapeutic target for anticancer therapy. Expert Opin Ther Targets 11: 81-90, 2007.

8. Folkman J: Fundamental concepts of the angiogenic process. Curr Mol Med 3: 643-651, 2003.

9. Carmeliet P and Jain RK: Angiogenesis in cancer and other diseases. Nature 407: 249-257, 2000.

10. Jain RK: Molecular regulation of vessel maturation. Nat Med 9: 685-693, 2003.

11. McMahon G: VEGF receptor signaling in tumor angiogenesis. Oncologist 5: 3-10, 2000.
12. Glade-Bender J, Kandel JJ and Yamashiro DJ: VEGF blocking therapy in the treatment of cancer. Expert Opin Biol Ther 3: 263-276, 2003.

13. Jain RK: Tumor angiogenesis and accessibility: role of vascular endothelial growth factor. Semin Oncol 29: 3-9, 2002.

14. Carmeliet P: VEGF as a key mediator of angiogenesis in cancer. Oncology 69: 4-10, 2005.

15. Cross MJ, Dixelius J, Matsumoto T and Claesson-Welsh L: VEGF-receptor signal transduction. Trends Biochem Sci 28 488-494, 2003.

16. Chakraborty G, Jain S and Kundu GC: Osteopontin promotes vascular endothelial growth factor-dependent breast tumor growth and angiogenesis via autocrine and paracrine mechanisms. Cancer Res 68: 152-161, 2008.

17. Shishodia S, Chaturvedi MM and Aggarwal BB: Role of curcumin in cancer therapy. Curr Probl Cancer 31: 243-305, 2007.

18. Aggarwal BB, Sundaram C, Malani N and Ichikawa H: Curcumin: the Indian solid gold. Adv Exp Med Biol 595: 1-75, 2007.

19. Arbiser JL, Klauber N, Rohan R, van Leeuwen R, Huang MT, Fisher C, Flynn E and Byers HR: Curcumin is an in vivo inhibitor of angiogenesis. Mol Med 4: 376-383, 1998.

20. Shao ZM, Shen ZZ, Liu CH, Sartippour MR, Go VL, Heber D and Nguyen M: Curcumin exerts multiple suppressive effects on human breast carcinoma cells. Int J Cancer 98: 234-240, 2002.

21. Dorai T and Aggarwal BB: Role of chemopreventive agents in cancer therapy. Cancer Lett 215: 129-140, 2004.

22. Aggarwal BB, Takada Y and Oommen OV: From chemoprevention to chemotherapy: common targets and common goals. Expert Opin Investig Drugs 13: 1327-1338, 2004.

23. Bachmeier BE, Mohrenz IV, Mirisola V, Schleicher E, Romeo F, Höhneke C, Jochum M, Nerlich AG and Pfeffer U: Curcumin down-regulates the inflammatory cytokines CXCL1 and -2 in breast cancer cells via NFkappaB. Carcinogenesis 29: 779-789, 2008.

24. Philip S and Kundu GC: Osteopontin induces nuclear factor kappa B-mediated promatrix metalloproteinase-2 activation through I kappa B alpha/IKK signaling pathways, and curcumin (diferulolylmethane) down-regulates these pathways. J Biol Chem 278: 14487-14497, 2003.

25. Philip S, Bulbule A and Kundu GC: Osteopontin stimulates tumor growth and activation of promatrix metalloproteinase- 2 through nuclear factor-kappa B-mediated induction of membrane type 1 matrix metalloproteinase in murine melanoma cells. J Biol Chem 276: 44926-44935, 2001.

26. Chakraborty G, Rangaswami H, Jain S and Kundu GC: Hypoxia regulates cross-talk between Syk and Lck leading to breast cancer progression and angiogenesis. J Biol Chem 281: 11322-11331, 2006.

27. Jain S, Chakraborty G and Kundu GC: The crucial role of cyclooxygenase-2 in osteopontin-induced protein kinase $\mathrm{C}$ alpha/ c-Src/IkappaB kinase alpha/beta-dependent prostate tumor progression and angiogenesis. Cancer Res. 66: 6638-6648, 2006.

28. Roybal CN, Yang S, Sun CW, Hurtado D, Vander Jagt DL, Townes TM and Abcouwer SF: Homocysteine increases the expression of vascular endothelial growth factor by a mechanism involving endoplasmic reticulum stress and transcription factor ATF4. J Biol Chem 279: 14844-14852, 2004.

29. Roybal CN, Hunsaker LA, Barbash O, Vander Jagt DL and Abcouwer SF: The oxidative stressor arsenite activates vascular endothelial growth factor mRNA transcription by an ATF4dependent mechanism. J Biol Chem 280: 20331-20339, 2005.

30. Chakraborty G, Jain S, Behera R, Ahmed M, Sharma P, Kumar V and Kundu GC: The multifaceted roles of osteopontin in cell signaling, tumor progression and angiogenesis. Curr Mol Med 6: 819-830, 2006.

31. Bellahcène A, Castronovo V, Ogbureke KU, Fisher LW and Fedarko NS: Small integrin-binding ligand N-linked glycoproteins (SIBLINGs): multifunctional proteins in cancer. Nat Rev Cancer 8: 212-226, 2008.

32. Weber GF: The metastasis gene osteopontin: a candidate target for cancer therapy. Biochim Biophys Acta 1552: 61-85, 2001.

33. Ruegg $\mathrm{C}$ and Mutter N: Anti-angiogenic therapies in cancer: achievements and open questions. Bull Cancer 94: 753-762, 2007. 